\title{
Higher angular momentum states of bottomonium in lattice NRQCD
}

\author{
Randy Lewis \\ Department of Physics and Astronomy, \\ York University, Toronto, Ontario, Canada MЗJ 1 P3 \\ R. M. Woloshyn \\ TRIUMF, 4004 Wesbrook Mall, Vancouver, \\ British Columbia, Canada V6T 2A3
}

\begin{abstract}
On a cubic lattice the zero-momentum meson states have one of 20 possible $\Lambda^{P C}$ combinations where $\Lambda$ labels the irreducible representation of the octahedral group. Each continuum bottomonium state with specific $J^{P C}$ quantum numbers is contained within one or more of the lattice $\Lambda^{P C}$ states. In this work, bottomonium quark-antiquark operators are constructed for all 20 lattice $\Lambda^{P C}$ combinations which allows many continuum high angular momentum states to be accessible as ground states of their associated lattice channels. From a dynamical simulation, realistic results are obtained for S-, P-, D-, F- and G-wave bottomonium states.
\end{abstract}




\section{MOTIVATION}

Although the lightest spin-triplet S-wave and P-wave bottomonia have been known experimentally for many years[1], the spin singlets have just been discovered in recent experiments[2 [5]. Beyond the $\mathrm{P}$ waves only the $\Upsilon\left(1^{3} D_{2}\right)$ has been observed so far[6, 7]. Meanwhile, additional resonances, which may have an interpretation as four-quark combinations, are being found with masses in the bottomonium region[8, 9]. Also, observation of the $\eta_{b}(2 S)$ has recently been claimed, though with a surprisingly small mass [10, 11].

Dynamical lattice QCD computations of the masses of S-wave and P-wave bottomonia, including radial excitations, appear in [12 16]. Although some of these publications also commented on D-wave states, the first lattice study of the full set of D-wave quantum numbers is very recent[17].

There are many important reasons for extending lattice studies to higher angular momentum states. D waves and $\mathrm{F}$ waves provide an opportunity to confirm and refine lattice methods, such as lattice NRQCD that uses a nonrelativistic expansion for the valence quarks [18]. Quantitative predictions for the masses can subsequently be tested experimentally, and comparison of reliable lattice results with today's phenomenological models can offer insights into various model assumptions. In particular, the spectrum as a function of orbital angular momentum compared to radial excitations depends on the spatial behavior of the quark-antiquark interaction. Extending the study of bottomonium to higher partial waves augments the information gained by the observation of radial excited states. The lightest G-wave bottomonium is expected to lie just below the $B \bar{B}$ threshold with $\mathrm{F}$ waves further below and $\mathrm{H}$ waves above[19], so these bottomonium channels offer an opportunity for using lattice QCD to study threshold issues in detail, and to confirm which side of threshold the $\mathrm{G}$ waves are on. Furthermore, knowledge of the bottomonium masses will be crucial for understanding the four-quark candidates and any other exotic hadrons that could be nearby. Eventually, radiative transitions among these states could be considered[20].

In general, a lattice operator will couple to all hadrons with the corresponding quantum numbers, but the lightest hadron will have the cleanest signal. Often the lightest hadron provides the only usable signal in a lattice study. As a result of cubic symmetry, there are just 20 mutually orthogonal $\Lambda^{P C}$ options available to lattice simulations. Specifically, the 20 options originate from the reduction of continuum angular momentum $J$ to the five lattice 
octahedral representations $\Lambda$, each with $P= \pm 1$ and $C= \pm 1$. All S-wave and P-wave bottomonium states are included in that list of 20 "ground states", along with just 10 higher waves. All other continuum bottomonium quantum numbers are only accessible as excited states in the 20 channels. As well, any hybrids, exotics, and multiquark states would also be included within the list of 20 possible lattice channels.

It is not known, a priori, which hadron is actually the lightest in a given $\Lambda^{P C}$ channel. For example, the quark model suggests that a $9^{+-}$bottomonium state will be the lightest in one of the 20 channels, but that same channel will couple to a $0^{+-}$exotic hadron if one exists. To discover which is the lighter state, it is necessary to perform an explicit lattice simulation and analysis. Identifying the lightest mass in each of the 20 channels is a natural starting point for lattice QCD explorations of bottomonia at higher angular momentum.

The specific contributions of the present work are twofold. First, a set of quark-antiquark creation operators for all 20 combinations of lattice quantum numbers is constructed and the lowest continuum angular momentum is identified. Secondly, numerical simulations are used to show that many of the higher angular momentum bottomonium states are accessible using present lattice QCD methods and existing gauge field configurations.

\section{BOTTOMONIUM OPERATORS FOR ALL LATTICE QUANTUM NUMBERS}

Conservation of angular momentum is a consequence of invariance under spatial rotations, and is realized as a tower of representations labeled by non-negative integers $J \in\{0,1,2, \ldots\}$. On a spatially cubic lattice, this symmetry is reduced to the five irreducible representations $\Lambda \in\left\{A_{1}, A_{2}, E, T_{1}, T_{2}\right\}$ of the octahedral group[21]. The well-known correspondence between $J$ and $\Lambda$ is given in Table I. In principle a sophisticated analysis of excited states in lattice data for each $\Lambda$ channel would allow the unambiguous identification of $J$ for each observed lattice state. However, experience from basic quantum mechanics and the quark model suggests that larger $J$ leads to larger bottomonium mass, so in practice it is simple to infer each $J$ value from a single channel (unless a non quark model hadron appears with a nearby mass).

Total angular momentum is the sum of orbital and spin components. The orbital an- 
Table I: The number of copies of each octahedral irreducible representation $\Lambda$, of dimension $d$, for the subduced continuum representation $J$.

\begin{tabular}{llllllllllll}
\hline \hline$\Lambda(d)$ & \multicolumn{8}{c}{$J$} \\
& 0 & 1 & 2 & 3 & 4 & 5 & 6 & 7 & 8 & 9 & $\cdots$ \\
\hline$A_{1}(1)$ & 1 & 0 & 0 & 0 & 1 & 0 & 1 & 0 & 1 & 1 & $\cdots$ \\
$A_{2}(1)$ & 0 & 0 & 0 & 1 & 0 & 0 & 1 & 1 & 0 & 1 & $\cdots$ \\
$E(2)$ & 0 & 0 & 1 & 0 & 1 & 1 & 1 & 1 & 2 & 1 & $\cdots$ \\
$T_{1}(3)$ & 0 & 1 & 0 & 1 & 1 & 2 & 1 & 2 & 2 & 3 & $\cdots$ \\
$T_{2}(3)$ & 0 & 0 & 1 & 1 & 1 & 1 & 2 & 2 & 2 & 2 & $\cdots$ \\
\hline \hline
\end{tabular}

gular momentum $L$ of each operator corresponds to the number of uncontracted ${ }^{1}$ spatial derivatives, but it is important to remember that $\Lambda$ (the lattice version of $J$ not $L$ ) is the conserved quantum number. An operator intended for a specific $L$ will couple to other $L$ values too, unless the fields are smeared in a way that purposefully eliminates that mixing.

One operator for each of the $20 \Lambda^{P C}$ channels is provided in Table II. They are written in terms of Pauli matrices $\sigma_{i}$ and the symmetric lattice covariant derivative $\Delta_{i}$ which appears beyond the $\mathrm{P}$ waves in the following forms:

$$
\begin{aligned}
D_{i j} & =\frac{1}{2}\left(\Delta_{i} \Delta_{j}+\Delta_{j} \Delta_{i}\right)-\frac{1}{3} \delta_{i j}\left(\Delta_{1}^{2}+\Delta_{2}^{2}+\Delta_{3}^{2}\right) \\
D_{i j k} & =\frac{1}{3 !}\left(\Delta_{i} \Delta_{j} \Delta_{k}+\text { permutations }\right) \\
D_{i j k l} & =\frac{1}{4 !}\left(\Delta_{i} \Delta_{j} \Delta_{k} \Delta_{l}+\text { permutations }\right) \\
D_{i j k l m} & =\frac{1}{5 !}\left(\Delta_{i} \Delta_{j} \Delta_{k} \Delta_{l} \Delta_{m}+\text { permutations }\right) \\
D_{i j k l m n} & =\frac{1}{6 !}\left(\Delta_{i} \Delta_{j} \Delta_{k} \Delta_{l} \Delta_{m} \Delta_{n}+\text { permutations }\right) \\
D_{i j k l m n o p q} & =\frac{1}{9 !}\left(\Delta_{i} \Delta_{j} \Delta_{k} \Delta_{l} \Delta_{m} \Delta_{n} \Delta_{o} \Delta_{p} \Delta_{q}+\text { permutations }\right) .
\end{aligned}
$$

The entries in Table I can be verified by using standard group theory methods to calculate multiplicities from the character of each conjugacy class, but an intuitive understanding can be obtained more readily. For example, the ${ }^{1} F_{3}$ operator is listed as $D_{123}$. Any spatial

\footnotetext{
${ }^{1}$ A lattice Laplacian, for example, does contain a Lorentz contraction and does not alter the orbital angular momentum.
} 
rotation of this operator on a lattice will reproduce the same operator except that its indices may be permuted and for certain rotations there will be an overall minus sign due to the vector nature of a spatial derivative. These spatial rotations have the properties of the one-dimensional representation $A_{2}$, including the overall minus signs for the appropriate rotations. $P$ and $C$ properties can also be verified.

For use with lattice NRQCD, each of the operators in Table II will be sandwiched between a bottom creation operator $\psi^{\dagger}(x)$ and an antibottom creation operator $\chi^{\dagger}(x)$, then summed spatially to produce a zero-momentum interpolating operator:

$$
\sum_{\vec{x}} \psi^{\dagger}(\vec{x}) \Omega \chi^{\dagger}(\vec{x})
$$

The $\psi^{\dagger}(x)$ and $\chi^{\dagger}(x)$ can contain internal summations as well, to represent spatial smearing. In our simulations, covariant derivatives act on the field to the right and not to the left. Charge conjugation is maintained exactly for all operators because of the sum over $\vec{x}$ in Eq. (17).

A table similar to Table II] appears in [22], but with a few notable differences. In [22] the authors listed only S, P and D waves, and their double derivative operator $\Delta_{i j}$ is not symmetrized in its indices, whereas we prefer to use a symmetric definition so exact symmetries are apparent. For the $E^{P C}$ channels, which are two-dimensional representations, the authors of [22] chose to list three nonindependent projections whereas we prefer to list the two independent projections. They also included three additional operators in the $T_{1}^{--}$ and $T_{2}^{--}$channels, intended to probe D-wave states which, however, are not expected to be ground states in their respective $\Lambda^{P C}$ channels; these operators are displayed in our Table III for convenient reference. For relativistic operators rather than NRQCD operators, as might be more appropriate for charmonium, see [23, 24].

Although the list of 20 quantum numbers $\Lambda^{P C}$ is complete, the operators listed for each are not unique. The operators displayed in Table【were chosen with standard bottomonium states in mind, so each contains one quark, one antiquark and $L$ derivatives. These operators will in general have some overlap with exotics, hybrids and multiquark states as well, but a search meant to target those states should construct other operators with the goal of having a larger overlap with the particular states of interest (see, for example, [24]).

The ${ }^{3} D_{1}$ meson does not appear in Table $\llbracket$ because it has the same $J^{P C}$ as the lighter ${ }^{3} S_{1}$ meson. Similarly, the ${ }^{3} F_{2}$ meson does not appear in Table $\llbracket$ because it has the same $J^{P C}$ as 
Table II: Creation operators $\Omega$ for each irreducible representation $\Lambda$ of the octahedral group with specified parity $P$ and charge conjugation $C$. Columns labeled $J^{P C}$ and ${ }^{2 S+1} L_{J}$ contain the quantum numbers of quark-antiquark states obtained using the standard relation of $S$ and $L$ with parity and charge conjugation.

\begin{tabular}{|c|c|c|}
\hline \multicolumn{3}{|c|}{$\Lambda^{P C} J^{P C 2 S+1} L_{J} \Omega$} \\
\hline$A_{1}^{-+} 0^{-+}$ & ${ }^{1} S_{0}$ & 1 \\
\hline$T_{1}^{--} 1^{--}$ & ${ }^{3} S_{1}$ & $\left\{\sigma_{1}, \sigma_{2}, \sigma_{3}\right\}$ \\
\hline$T_{1}^{+-} 1^{+-}$ & ${ }^{1} P_{1}$ & $\left\{\Delta_{1}, \Delta_{2}, \Delta_{3}\right\}$ \\
\hline$A_{1}^{++} 0^{++}$ & ${ }^{3} P_{0}$ & $\Delta_{1} \sigma_{1}+\Delta_{2} \sigma_{2}+\Delta_{3} \sigma_{3}$ \\
\hline$T_{1}^{++} 1^{++}$ & ${ }^{3} P_{1}$ & $\left\{\Delta_{2} \sigma_{3}-\Delta_{3} \sigma_{2}, \Delta_{3} \sigma_{1}-\Delta_{1} \sigma_{3}, \Delta_{1} \sigma_{2}-\Delta_{2} \sigma_{1}\right\}$ \\
\hline$E^{++} 2^{++}$ & ${ }^{3} P_{2}$ & $\left\{\left(\Delta_{1} \sigma_{1}-\Delta_{2} \sigma_{2}\right) / \sqrt{2},\left(\Delta_{1} \sigma_{1}+\Delta_{2} \sigma_{2}-2 \Delta_{3} \sigma_{3}\right) / \sqrt{6}\right\}$ \\
\hline$T_{2}^{++} 2^{++}$ & ${ }^{3} P_{2}$ & $\left\{\Delta_{2} \sigma_{3}+\Delta_{3} \sigma_{2}, \Delta_{3} \sigma_{1}+\Delta_{1} \sigma_{3}, \Delta_{1} \sigma_{2}+\Delta_{2} \sigma_{1}\right\}$ \\
\hline$E^{-+} 2^{-+}$ & ${ }^{1} D_{2}$ & $\left\{\left(D_{11}-D_{22}\right) / \sqrt{2},\left(D_{11}+D_{22}-2 D_{33}\right) / \sqrt{6}\right\}$ \\
\hline$T_{2}^{-+} 2^{-+}$ & ${ }^{1} D_{2}$ & $\left\{D_{23}, D_{31}, D_{12}\right\}$ \\
\hline$E^{--} 2^{--}$ & ${ }^{3} D_{2}$ & $\left\{\left(D_{23} \sigma_{1}-D_{13} \sigma_{2}\right) / \sqrt{2},\left(D_{23} \sigma_{1}+D_{31} \sigma_{2}-2 D_{12} \sigma_{3}\right) / \sqrt{6}\right\}$ \\
\hline$T_{2}^{--} 2^{--}$ & ${ }^{3} D_{2}$ & $\begin{array}{l}\left\{\left(D_{22}-D_{33}\right) \sigma_{1}+D_{13} \sigma_{3}-D_{12} \sigma_{2},\left(D_{33}-D_{11}\right) \sigma_{2}+D_{21} \sigma_{1}-D_{23} \sigma_{3}\right. \\
\left.\left(D_{11}-D_{22}\right) \sigma_{3}+D_{32} \sigma_{2}-D_{31} \sigma_{1}\right\}\end{array}$ \\
\hline$A_{2}^{--} 3^{--}$ & ${ }^{3} D_{3}$ & $D_{12} \sigma_{3}+D_{23} \sigma_{1}+D_{31} \sigma_{2}$ \\
\hline$A_{2}^{+-} 3^{+-}$ & ${ }^{1} F_{3}$ & $D_{123}$ \\
\hline$T_{2}^{+-} 3^{+-}$ & ${ }^{1} F_{3}$ & $\left\{D_{122}-D_{133}, D_{233}-D_{211}, D_{311}-D_{322}\right\}$ \\
\hline$A_{2}^{++} 3^{++}$ & ${ }^{3} F_{3}$ & $\left(D_{221}-D_{331}\right) \sigma_{1}+\left(D_{332}-D_{112}\right) \sigma_{2}+\left(D_{113}-D_{223}\right) \sigma_{3}$ \\
\hline$T_{1}^{-+} 4^{-+}$ & ${ }^{1} G_{4}$ & $\left\{D_{2223}-D_{3332}, D_{3331}-D_{1113}, D_{1112}-D_{2221}\right\}$ \\
\hline$A_{1}^{--} 4^{--}$ & ${ }^{3} G_{4}$ & $\left(D_{2223}-D_{3332}\right) \sigma_{1}+\left(D_{3331}-D_{1113}\right) \sigma_{2}+\left(D_{1112}-D_{2221}\right) \sigma_{3}$ \\
\hline$E^{+-} 5^{+-}$ & ${ }^{1} H_{5}$ & $\left\{\left(D_{23111}-D_{13222}\right) / \sqrt{2},\left(D_{23111}+D_{13222}-2 D_{12333}\right) / \sqrt{6}\right\}$ \\
\hline$A_{2}^{-+} 6^{-+}$ & ${ }^{1} I_{6}$ & $D_{112222}+D_{223333}+D_{331111}-D_{221111}-D_{332222}-D_{113333}$ \\
\hline$A_{1}^{+-} 9^{+-}$ & ${ }^{1} L_{9}$ & $D_{122233333}+D_{233311111}+D_{311122222}-D_{133322222}-D_{211133333}-D_{322211111}$ \\
\hline
\end{tabular}

the lighter ${ }^{3} P_{2}$ meson. Neither of those omissions is a consequence of lattice discretization, since even the continuum quantum numbers are shared. On the other hand, the absence of the ${ }^{3} F_{4}$ meson from Table $\llbracket$ is a consequence of lattice discretization: its continuum quantum numbers $\left(4^{++}\right)$are not shared by any lighter state, but its lattice quantum numbers $\left(A_{1}^{++}\right.$, 
Table III: Creation operators $\Omega$ that duplicate some quantum numbers from Table II but could be useful for studies of the D-wave spectrum. They were first listed in [22].

\begin{tabular}{lll}
\hline \hline$\Lambda^{P C} J^{P C}{ }^{2 S+1} L_{J}$ & $\Omega$ \\
\hline$T_{1}^{--} 1^{--}$ & ${ }^{3} D_{1}$ & $\left\{D_{11} \sigma_{1}+D_{12} \sigma_{2}+D_{13} \sigma_{3}, D_{21} \sigma_{1}+D_{22} \sigma_{2}+D_{23} \sigma_{3}, D_{31} \sigma_{1}+D_{32} \sigma_{2}+D_{33} \sigma_{3}\right\}$ \\
$T_{1}^{--} 3^{--}$ & ${ }^{3} D_{3}$ & $\left\{3 D_{11} \sigma_{1}-2 D_{12} \sigma_{2}-2 D_{13} \sigma_{3}, 3 D_{22} \sigma_{2}-2 D_{23} \sigma_{3}-2 D_{21} \sigma_{1}\right.$, \\
& & $\left.3 D_{33} \sigma_{3}-2 D_{31} \sigma_{1}-2 D_{32} \sigma_{2}\right\}$ \\
$T_{2}^{--} 3^{--}$ & ${ }^{3} D_{3}$ & $\left\{\left(D_{22}-D_{33}\right) \sigma_{1}+2 D_{12} \sigma_{2}-2 D_{13} \sigma_{3},\left(D_{33}-D_{11}\right) \sigma_{2}+2 D_{23} \sigma_{3}-2 D_{21} \sigma_{1}\right.$, \\
& & $\left.\left(D_{11}-D_{22}\right) \sigma_{3}+2 D_{31} \sigma_{1}-2 D_{32} \sigma_{2}\right\}$ \\
\hline \hline
\end{tabular}

$E^{++}, T_{1}^{++}, T_{2}^{++}$) are all shared by the ${ }^{3} P_{J}$ mesons. Therefore lattice signals of the ${ }^{3} D_{1}$, ${ }^{3} F_{2},{ }^{3} F_{4}$ and many similar cases must be sought as excited states in the corresponding $\Lambda^{P C}$ channels, unless operators can be tuned carefully enough to remove any coupling to the lighter mesons in each channel. Analysis of excited states in any $\Lambda^{P C}$ channel is beyond the scope of the present work with one exception: we will address the $\eta_{b}(2 S)$ because of the present experimental situation[10,11].

\section{LATTICE SIMULATIONS}

All numerical simulations performed for this work utilize a $2+1$-flavor dynamical gauge field ensemble generated by the PACS-CS Collaboration[25] and made available through the Japan Lattice Data Grid[26] as part of the International Lattice Data Grid[27]. This ensemble uses the Iwasaki gauge action and the nonperturbatively tuned clover action for up, down, and strange quarks. The light quark (up and down) masses are such that the pion mass is $156 \mathrm{MeV} / c^{2}$, i.e. near the physical value. There are 198 configurations with lattice volume $32^{3} \times 64$. The four input parameters, along with four basic output quantities, are displayed in Table IV. Not provided by the PACS-CS Collaboration but needed for the present work is the mean link in Landau gauge that was computed[20] to be $u_{L}=0.8463$.

The bottom quark and antiquark are described by the lattice NRQCD Hamiltonian[18] omitting terms at $O\left(v^{6}\right)$ and beyond. Specifically, the Hamiltonian from the Appendix of [28] is used with $c_{i}=1$ for $i \leq 6$ and $c_{i}=0$ for $i \geq 7$, with tadpole factor $U_{0}=u_{L}=0.8463$ and with stability parameter $n=4$. 
Table IV: Input parameters for the ensemble of gauge configurations generated by the PACS-CS Collaboration[25]. Some standard output quantities provided by PACS-CS are also listed.

\begin{tabular}{ll}
\hline \hline Inputs & Standard outputs \\
\hline$\beta=1.90$, & $a=0.0907(13) \mathrm{fm}$ \\
$\kappa_{u d}=0.13781$, & $m_{\pi}=156(7) \mathrm{MeV} / c^{2}$ \\
$\kappa_{s}=0.13640$, & $m_{K}=554(8) \mathrm{MeV} / c^{2}$ \\
$c_{S W}=1.715$, & $\langle\square\rangle=0.570870(5)$ \\
\hline \hline
\end{tabular}

In previous work[20] the bottom quark bare mass was tuned to match the experimental $\eta_{b}(1 S)$ mass, with result $M_{b}=1.945$. However, a recent measurement by the Belle Collaboration[29] is significantly larger than the $\eta_{b}(1 S)$ mass reported by previous experiments[1]. Tuning to the Belle result would require a slight increase in the bottom quark bare mass of lattice NRQCD. To avoid this unresolved issue, it is preferable to use the well-established $\Upsilon(1 S)$ mass[1] for tuning instead. This also leads to a slight increase in the bare mass parameter relative to our previous work[20], and for the present project we use $M_{b}=1.95$.

To reduce statistical fluctuations, it would be optimal to average over results obtained from putting the creation operator at each lattice site. A practical implementation of this general idea is employed here: heavy quark propagators are constructed from a random-U(1) wall source[30] spanning all spatial lattice sites at a fixed Euclidean time. A further reduction of statistical fluctuations can be achieved by selecting another Euclidean time on the same gauge configuration, separately inserting a random-U(1) wall source there, and averaging the two correlation functions on that configuration. For the present work, averages are taken over 64 separate calculations of all correlation functions per configuration, corresponding to a random-U(1) wall source at each Euclidean time on the $32^{3} \times 64$ lattice.

Excellent ground state signals are obtained for $\mathrm{S}$ and $\mathrm{P}$ waves with a single correlation function using a local operator. As one goes to higher waves, simulation energies increase and correlation functions decrease more rapidly and become noisier due to the presence of more derivatives in the operators. It is advantageous to utilize more correlators in each channel and to smear the operators to enhance the ground state contribution at smaller time separations. Beyond the $\mathrm{P}$ waves, gauge-invariant smearing[31] is applied to the bottom 
quark operator,

$$
\psi(x) \rightarrow\left(1+0.15 \Delta^{2}\right)^{8 s} \psi(x) .
$$

Three options are considered: $s=0$ (local), $s=1$ (smeared), and $s=2$ (doubly smeared). In every case the antiquark field $\chi(x)$ remains unsmeared.

With local, smeared and doubly smeared operators available for both creation $(s)$ and annihilation $\left(s^{\prime}\right)$, each $\Lambda^{P C}$ has nine correlation functions to be analyzed simultaneously. The nominal fit function is

$$
g_{s, s^{\prime}}\left(t-t_{0}\right)=\sum_{n=1}^{N} f_{s}(n) f_{s^{\prime}}(n) e^{-E_{n}\left(t-t_{0}\right)}
$$

where the number of exponentials $N$ is increased until the $\chi^{2} /$ d.o.f. is unity or less. $E_{n}$ denotes the simulation energy for the $n$th meson while $f_{s}(n)$ and $f_{s^{\prime}}(n)$ denote the coupling of the $n$th meson to the creation and annihilation operators respectively.

In practice, the lattice data cannot always resolve individual higher-energy states and that requires a generalization of Eq. (9). As a simple example consider two operators, $A$ and $B$, that each couple to two physical mesons having energies $E_{1}$ and $E_{2}$. Suppose these energies are unequal but not distinguishable with the numerical precision of the available lattice data, so they get represented by a single exponential during the fitting process, $E_{1} \approx E_{2} \approx E_{\text {fit }}$. The nominal fit function (with $t_{0}=0$ ) would give

$$
\begin{aligned}
\left\langle 0\left|A A^{\dagger}\right| 0\right\rangle & =\left[f_{A}(1)\right]^{2} e^{-E_{1} t}+\left[f_{A}(2)\right]^{2} e^{-E_{2} t} \approx\left(\left[f_{A}(1)\right]^{2}+\left[f_{A}(2)\right]^{2}\right) e^{-E_{\mathrm{fit}} t} \\
\left\langle 0\left|B B^{\dagger}\right| 0\right\rangle & =\left[f_{B}(1)\right]^{2} e^{-E_{1} t}+\left[f_{B}(2)\right]^{2} e^{-E_{2} t} \approx\left(\left[f_{B}(1)\right]^{2}+\left[f_{B}(2)\right]^{2}\right) e^{-E_{\mathrm{fit}} t} \\
\left\langle 0\left|B A^{\dagger}\right| 0\right\rangle & =f_{A}(1) f_{B}(1) e^{-E_{1} t}+f_{A}(2) f_{B}(2) e^{-E_{2} t} \approx\left(f_{A}(1) f_{B}(1)+f_{A}(2) f_{B}(2)\right) e^{-E_{\mathrm{fit}} t} .
\end{aligned}
$$

The three coefficients of $e^{-E_{1} t}$ contain only two degrees of freedom as assumed in Eq. (9). The same is true for the coefficients of $e^{-E_{2} t}$. However, the three coefficients of $e^{-E_{\mathrm{fit}} t}$ do not contain just two degrees of freedom. To account for this potential inability of lattice data to resolve excited states, Eq. (9) can be generalized to

$$
g_{s, s^{\prime}}\left(t-t_{0}\right)=\sum_{n=1}^{N^{\prime}} f_{s}(n) f_{s^{\prime}}(n) e^{-E_{n}\left(t-t_{0}\right)}+\sum_{n=N^{\prime}+1}^{N} f_{s, s^{\prime}}(n) e^{-E_{n}\left(t-t_{0}\right)}
$$

which says the lowest $N^{\prime}$ states are resolved individually but higher states may not be. Confident extraction of the ground-state energy requires $N^{\prime}>1$, which is satisfied easily by all of the data discussed in this paper. 
The methods described above are sufficient to permit explorations in the S, P, D, F, G, H and I channels as presented in the following section, with reasonably precise results obtained in the S, P and D channels. Since F and G waves have not been discussed in previous lattice simulations but are expected to lie below the $B \bar{B}$ threshold[19], we focus particular attention on the $\mathrm{F}$ - and $\mathrm{G}$-wave channels by implementing, in addition to the methods described above, stout spatial links $\widetilde{U}$ as proposed in [32]:

$$
\begin{aligned}
& U \rightarrow U^{(1)} \rightarrow U^{(2)} \rightarrow \cdots \rightarrow U^{\left(n_{\rho}\right)} \equiv \widetilde{U}, \\
& U_{\mu}^{(n+1)}(x)=\exp \left(i Q_{\mu}^{(n)}(x)\right) U_{\mu}^{(n)}(x),
\end{aligned}
$$

where $Q_{\mu}^{(n)}(x)$ contains a product of gauge links weighted by a parameter $\rho$. [For the precise definition of $Q_{\mu}^{(n)}(x)$, see [32].] Our F- and G-wave simulations use $\rho=0.2$ and $n_{\rho}=16$ for source and sink operators, but no stouting within the NRQCD propagator.

Lattice simulations involve both systematic and statistical errors. In the present context, systematic errors arise from the neglect of $O\left(v^{6}\right)$ terms in the NRQCD Hamiltonian, the neglect of $O\left(\alpha_{s}\right)$ corrections to the NRQCD coefficients $c_{i}$, the nonzero lattice spacing, the finite lattice volume, and up and down quark masses that are slightly larger than their physical values. Although we do not have any quantitative means to determine systematic errors for ourselves, the authors of [12] studied bottomonium with the same truncation of NRQCD terms and tadpole-improved tree-level $c_{i}$ definitions on lattices having discretizations and volumes similar to ours, though their up and down quarks were significantly heavier than ours. The thorough discussion in [12] concluded that systematic errors in typical $\Upsilon$ mass splittings are at the few-percent level. We take this as a generic expectation for our systematic errors too, though only statistical errors will be reported below. Interestingly, the statistical errors will turn out to be at the few-percent level also.

\section{LATTICE RESULTS}

To provide a visual impression of the quality of lattice data, sample D-wave and F-wave correlation functions are displayed in Figs. 1 and 2 respectively. Numerical results for the fits are listed in Table $\mathrm{V}$. Because the smearing parameters were not optimized individually for each operator, two of the fits do not benefit from using all nine combinations of source and sink smearings; Table $\mathrm{V}$ records the number of combinations used in each case. Fits 


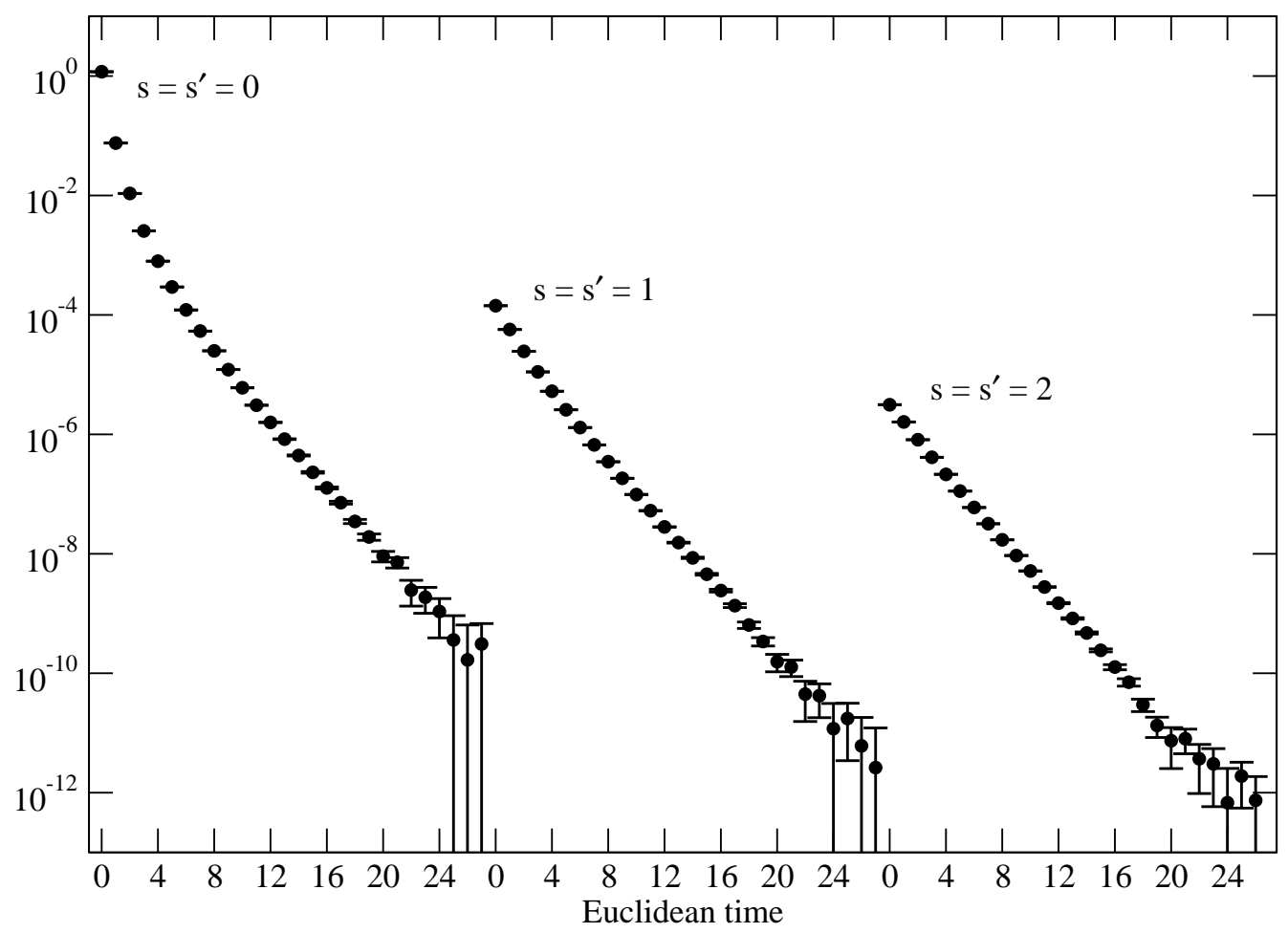

Figure 1: Correlation functions for $E^{--}$with equal smearing at source and sink. The lightest meson in this channel is ${ }^{3} D_{2}$ bottomonium.

employ the standard correlated $\chi^{2}$ method[33]. With a source at Euclidean time $t_{0}$, the Sand $\mathrm{P}$-wave fits include $1 \leq t-t_{0} \leq 31$, D-wave fits use $1 \leq t-t_{0} \leq 27$, and all higher-wave fits use $1 \leq t-t_{0} \leq 23$. The conversion from the lattice simulation energy $E_{\text {sim }}$ to a meson mass in physical units (also shown in Table $\mathrm{V}$ ) is accomplished by recalling that $M_{b}$ was tuned to the physical $\Upsilon(1 S)$ mass. Explicitly,

$$
M c^{2}=M\left(\Upsilon(1 S)_{\text {experiment }}\right) c^{2}+\frac{\hbar c}{a}\left(E_{\text {sim }}-E_{\text {sim }}(\Upsilon(1 S))\right)
$$

where the lattice spacing, $a$, is taken from Table IV.

The $\eta_{b}(1 S)$ mass in Table $\mathrm{V}$ is consistent with the Belle measurement and larger than the PDG average:

$$
M\left(\eta_{b}(1 S)\right)= \begin{cases}9390.9 \pm 2.8 \mathrm{MeV} / c^{2}, & \text { PDG average[1] } \\ 9401.0 \pm 1.9_{-2.4}^{+1.4} \mathrm{MeV} / c^{2}, & \text { Belle[29] } \\ 9404.0 \pm 0.8 \mathrm{MeV} / c^{2}, & \text { this work, statistical errors only. }\end{cases}
$$

It should be noted that this lattice result is consistent with some lattice determinations that predated the Belle measurement[12, 13, 20]. Studies of specific aspects of this issue are also 


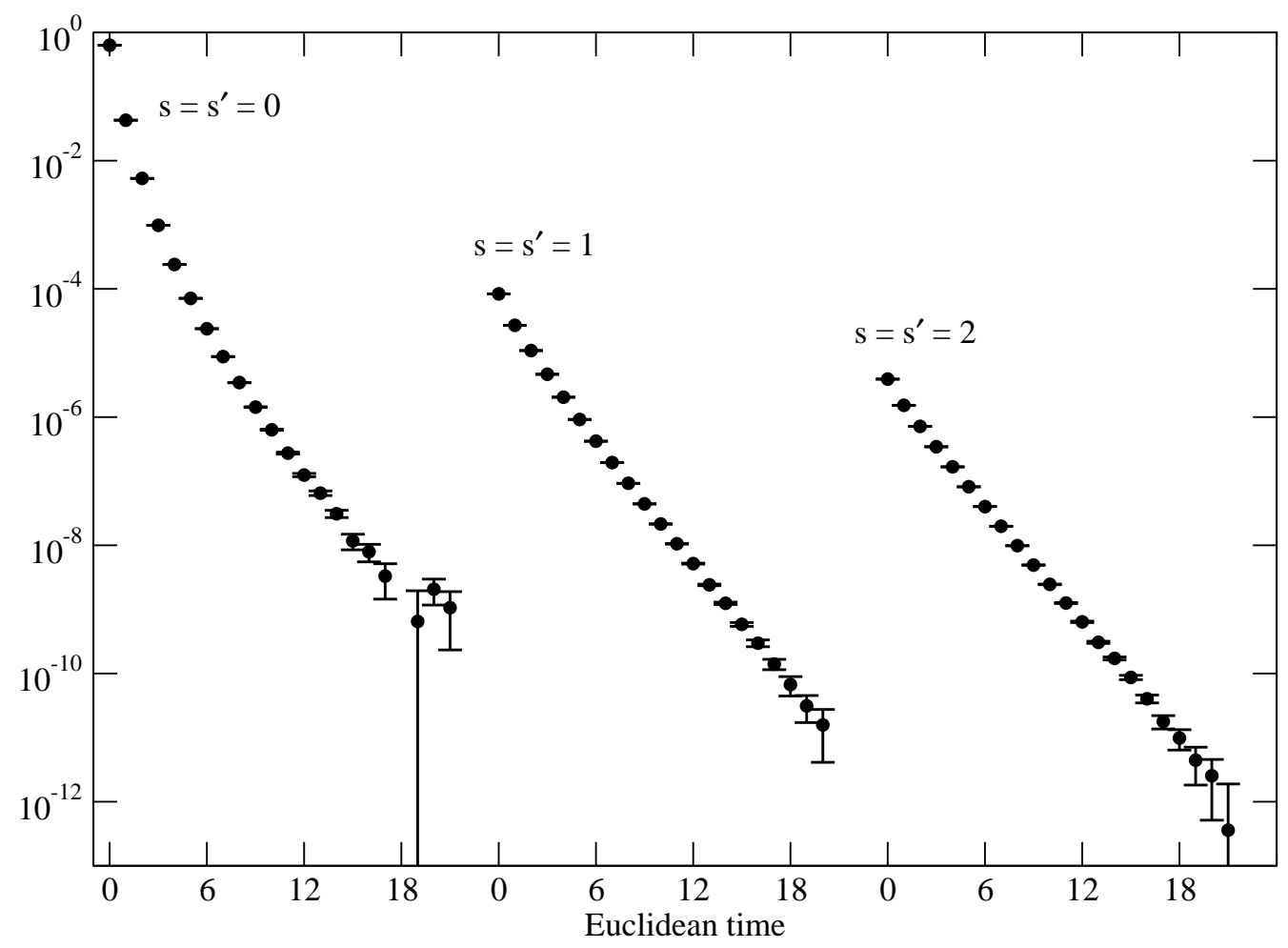

Figure 2: Correlation functions for $T_{2}^{+-}$with equal smearing at source sink. Stout links are used at source and sink as well. The lightest meson in this channel is ${ }^{1} F_{3}$ bottomonium.

available in [15, 34 ].

The $\mathrm{P}$-wave and $\mathrm{D}$-wave states are in reasonable agreement with the available experimental measurements, but splittings among D waves are not resolved, in contrast to [17]. The three F-wave results from Table V,

$$
\begin{aligned}
& M\left({ }^{1} F_{3}\right)=\left\{\begin{array}{l}
10.369 \pm 0.017 \mathrm{GeV} / c^{2}, \\
10.383 \pm 0.016 \mathrm{GeV} / c^{2}
\end{array}\right. \\
& M\left({ }^{3} F_{3}\right)=10.367 \pm 0.018 \mathrm{GeV} / c^{2},
\end{aligned}
$$

are in excellent agreement with a model prediction of $10.37 \mathrm{GeV} / c^{2}[19]$. The two G-wave results from Table $\mathrm{V}$,

$$
\begin{aligned}
& M\left({ }^{1} G_{4}\right)=10.581 \pm 0.017 \mathrm{GeV} / c^{2} \\
& M\left({ }^{3} G_{4}\right)=10.587 \pm 0.018 \mathrm{GeV} / c^{2}
\end{aligned}
$$

are about one standard deviation above the experimental $B \bar{B}$ threshold at $10.56 \mathrm{GeV} / c^{2}$. Recalling that the quoted lattice uncertainties are statistical only, our results are also rea- 
Table V: Simulation energies and resulting bottomonium masses obtained from fits to lattice data. Also shown are the number of correlators (i.e. smearing combinations) used in the fits and the number of exponentials needed to reduce the $\chi^{2} /$ d.o.f. to unity or less. All operators are from Table I except the one labeled $T_{2}^{--}\left({ }^{3} D_{3}\right)$ which is from Table III. Errors are statistical only.

\begin{tabular}{ccclc}
\hline \hline$\Lambda^{P C}$ & $n_{\text {corr }}$ & $n_{\text {exp }}$ & $E_{\text {sim }}$ & mass $\left[\mathrm{GeV} / c^{2}\right]$ \\
\hline$A_{1}^{-+}$ & 1 & 4 & $0.23420(6)$ & $9.4040(8)$ \\
$T_{1}^{--}$ & 1 & 4 & $0.25993(7)$ & 9.460 \\
$T_{1}^{+-}$ & 1 & 4 & $0.451(4)$ & $9.876(11)$ \\
$A_{1}^{++}$ & 1 & 4 & $0.438(4)$ & $9.847(11)$ \\
$T_{1}^{++}$ & 1 & 4 & $0.449(4)$ & $9.871(11)$ \\
$E^{++}$ & 1 & 4 & $0.457(7)$ & $9.889(17)$ \\
$T_{2}^{++}$ & 1 & 4 & $0.454(7)$ & $9.882(17)$ \\
$E^{-+}$ & 9 & 5 & $0.591(5)$ & $10.180(16)$ \\
$T_{2}^{-+}$ & 9 & 7 & $0.599(4)$ & $10.198(14)$ \\
$E^{--}$ & 9 & 6 & $0.599(4)$ & $10.198(14)$ \\
$T_{2}^{--}$ & 9 & 6 & $0.588(3)$ & $10.174(13)$ \\
$A_{2}^{--}$ & 9 & 6 & $0.600(3)$ & $10.200(13)$ \\
$T_{2}^{--}\left({ }^{3} D_{3}\right)$ & 9 & 6 & $0.601(3)$ & $10.202(13)$ \\
$T_{2}^{+-}$ & 9 & 6 & $0.684(4)$ & $10.369(17)$ \\
$A_{2}^{+-}$ & 9 & 5 & $0.690(3)$ & $10.383(16)$ \\
$A_{2}^{++}$ & 9 & 5 & $0.683(5)$ & $10.367(18)$ \\
$T_{1}^{-+}$ & 6 & 4 & $0.775(1)$ & $10.581(17)$ \\
$A_{1}^{--}$ & 6 & 4 & $0.778(3)$ & $10.587(18)$ \\
\hline \hline & & & & \\
\hline
\end{tabular}

sonably close to the model prediction of $10.52 \mathrm{GeV} / c^{2}[19]$ (which, however, lies below the $B \bar{B}$ threshold).

Fits to lattice data in $\mathrm{H}$-wave and I-wave channels could only support three or four exponentials, had no stout links, and lay above the $B \bar{B}$ threshold, so meaningful mass predictions were not obtained. We did not attempt an L-wave simulation.

The behavior of orbital excitations compared to radial excitations depends on the form of the potential. Figure 3 shows the $q \bar{q}$ potential used in the quark model of Godfrey 


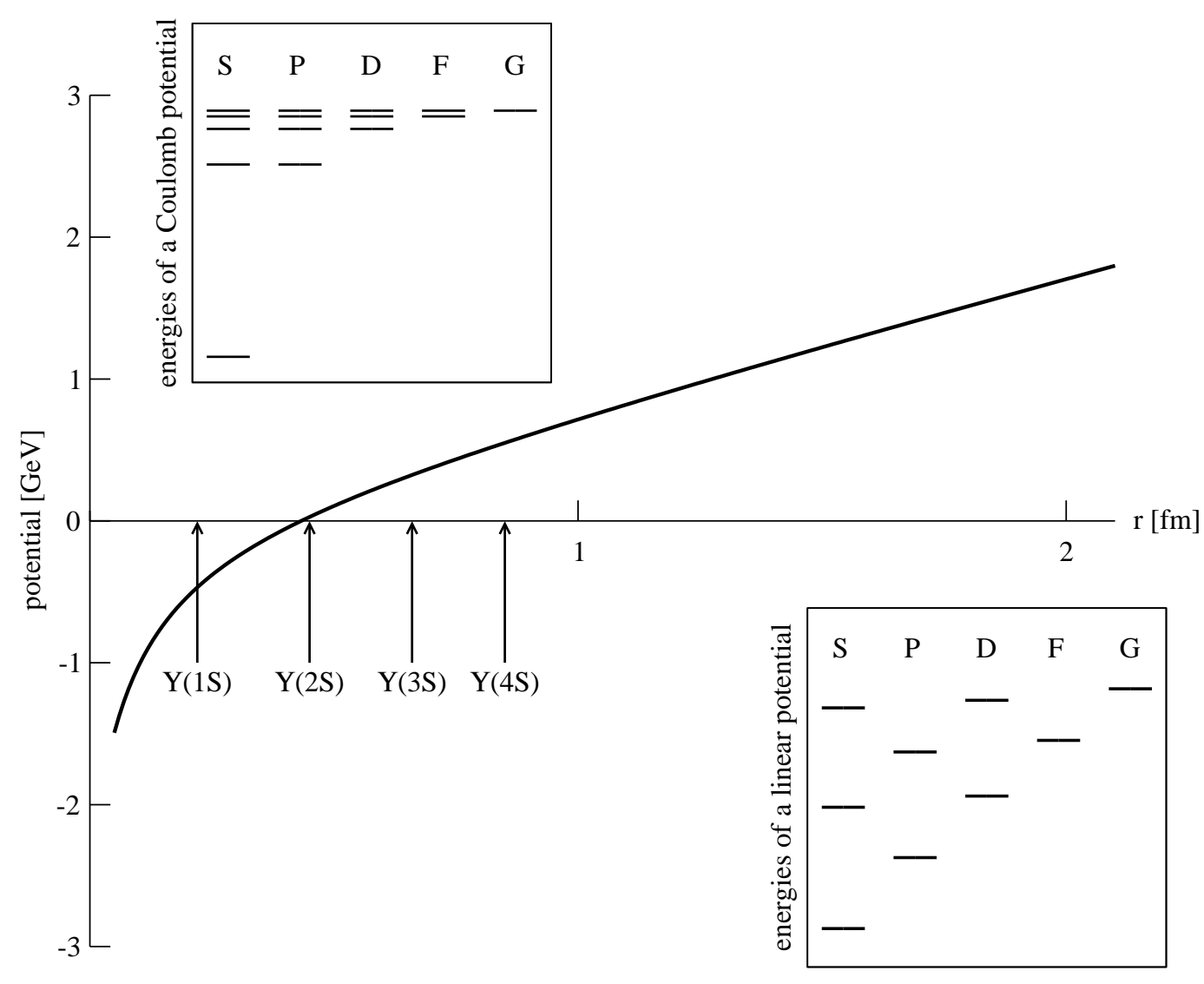

Figure 3: Main graph: The $q \bar{q}$ potential for mesons in the relativized quark model of Godfrey and Isgur[35]; see their Fig. 12. Upper inset: The spectrum of low-lying energy levels from a simple Coulomb potential. Lower inset: The spectrum of low-lying energy levels from a simple linear potential.

and Isgur[35], along with their determination of the location of some $\Upsilon$ excitations in that potential. The $\Upsilon(1 S)$ is in the Coulomb regime but higher radial excitations move into the transition region between Coulomb and linear. The insets in Fig. 3 show radial and orbital excitations in the simple cases of purely Coulomb and purely linear potentials. In calculations and from experimental data one finds that the ground-state P-wave states have masses not very different from the first S-wave radial excitations. There is at least some resemblance to the expectation from a Coulomb-like potential. The situation changes as angular momentum is increased. At $\mathrm{G}$ waves the ground state would be comparable to the $\Upsilon(5 S)$ in the Coulomb limit but comparable to $\Upsilon(3 S)$ in the linear limit. The lattice spectrum from the present work is displayed in Fig. 4 , where the $G$ wave is seen to be 


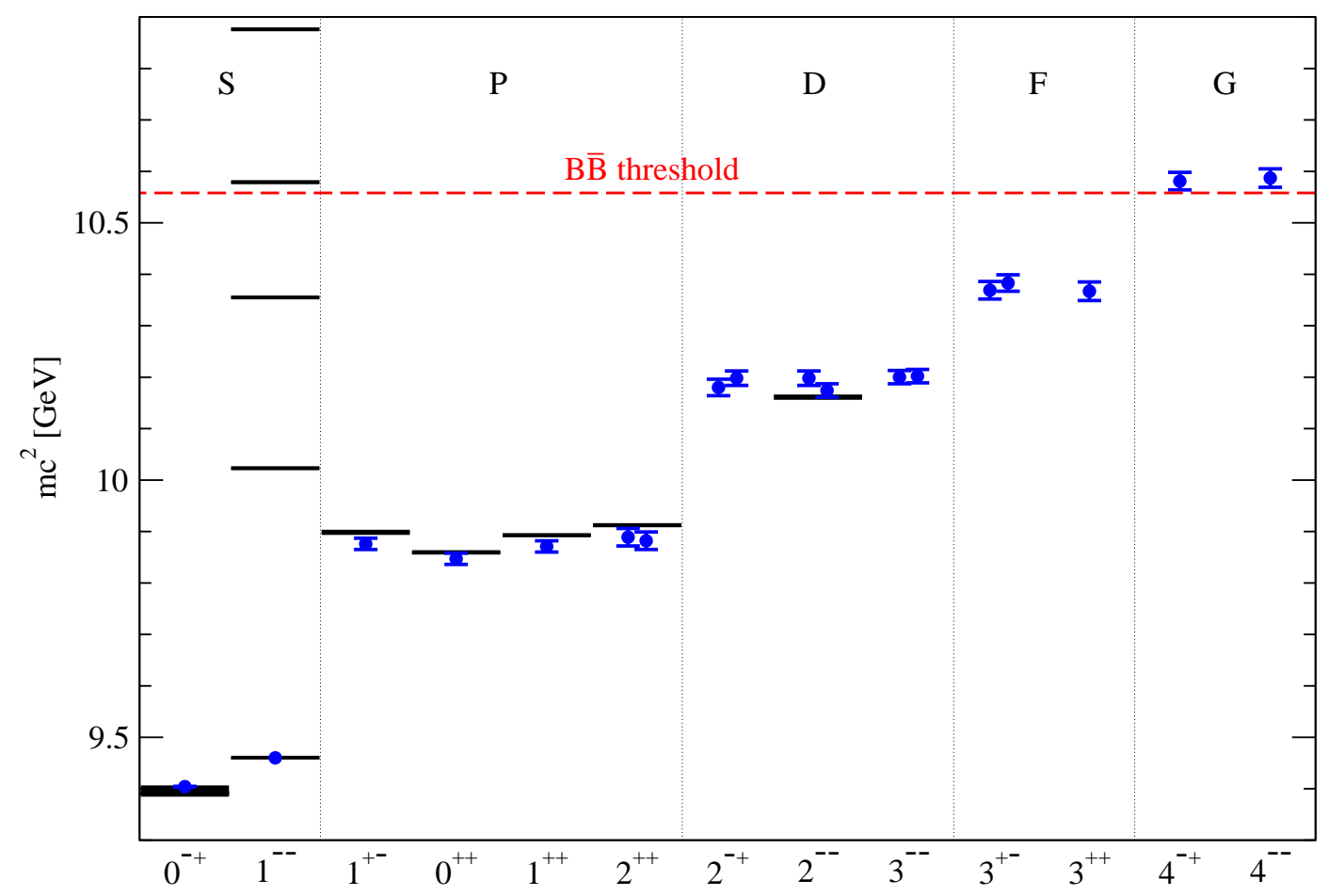

Figure 4: Lattice results for the bottomonium masses (data points with statistical error bars) compared with experimental data[1] (horizontal lines). Radially excited $\Upsilon$ masses are shown for comparison to orbital excitations.

comparable to the $\Upsilon(4 S)$ mass, i.e. intermediate to the Coulomb and linear cases. For the lattice results, the $G-F$ splitting is comparable to the $F-D$ splitting and thus more reminiscent of a linear-dominated potential than a Coulomb-dominated potential. Perhaps the most important conclusion about $\mathrm{G}$ waves in this work is that statistical errors are small enough to warrant future systematic lattice study to determine, for example, whether the $\mathrm{G}$ waves lie above or below threshold.

Although this work has focused exclusively on the ground state masses for each $\Lambda^{P C}$, excited state masses were obtained during the fitting process. In light of the present experimental situation[10, 11], the $\eta_{b}(2 S)$ is of particular interest. Our present work relies on local operators for the $\mathrm{S}$ waves, but our previous work[20] included smearing and thus produced a more precise result for the $\eta_{b}(2 S)$ mass. Since it was not reported explicitly in that publication, we include it here:

$$
M(\Upsilon(2 S))-M\left(\eta_{b}(2 S)\right)=24 \pm 3 \mathrm{MeV} / c^{2}
$$

where the error is statistical only. This hyperfine splitting is significantly smaller than the 
recently announced experimental measurement[10, 11], $M(\Upsilon(2 S))-M\left(\eta_{b}(2 S)\right)=48.7 \pm 2.7$ $\mathrm{MeV} / \mathrm{c}^{2}$, but our result is consistent with other lattice predictions[12, 14-16] as tabulated in [10].

\section{CONCLUSIONS}

The study of bottomonium at increasing angular momentum provides knowledge that augments that obtained from the spectrum of radial excitations. In this paper we provide a set of operators in lattice NRQCD for higher angular momentum bottomonium states and explore the feasibility of their use in numerical simulations.

A set of quark-antiquark operators for all lattice irreducible representations, $\Lambda^{P C}$, has been given in Table III. In principle, this set of operators allows for bottomonium states with 16 distinct continuum $J^{P C}$ combinations to be described as ground states in their respective lattice channels. All the $\mathrm{S}$ - and $\mathrm{P}$-wave states are in this set. However, for higher angular momentum, some continuum $J^{P C}$ combinations would only appear in $\Lambda^{P C}$ channels where a lower angular momentum state is present. These states would have to be extracted from lattice simulations as excited states. Since ground state energies are much easier to determine from lattice simulations, the states described by the operators in Table II] are a natural starting point for numerical studies of the high angular momentum states of bottomonium.

Our numerical simulations produced clear signals for S-, P-, D-, F- and G-wave states as displayed in Fig. 4. These are the first lattice simulations of F-wave and G-wave bottomonium, and they are near quark model expectations. The G waves are very close to the physical $B \bar{B}$ threshold, and statistical errors in the lattice simulation are small enough to encourage future studies of the systematic errors so that lattice methods can ultimately predict with confidence whether the $\mathrm{G}$ waves lie above or below threshold. Table II contains ground-state operators for H, I and L waves also, though these will be significantly above the $B \bar{B}$ threshold. 


\section{Acknowledgments}

We are grateful to the PACS-CS Collaboration for making their dynamical gauge field configurations available. R.L. thanks the ECT* in Trento for support, and the organizers of "Beautiful Mesons and Baryons on the Lattice" for the opportunity to participate while this research was in its final stages. The work was supported in part by the Natural Sciences and Engineering Research Council (NSERC) of Canada, and by computing resources of the Shared Hierarchical Academic Research Computing Network (SHARCNET) [36].

[1] K. Nakamura et al. (Particle Data Group), J. Phys. G 37, 075021 (2010) and 2011 partial update for the 2012 edition, http://pdg.lbl.gov

[2] B. Aubert et al. (BABAR Collaboration), Phys. Rev. Lett. 101, 071801 (2008); B. Aubert et al. (BABAR Collaboration), ibid. 102, 029901(E) (2009).

[3] B. Aubert et al. (BABAR Collaboration), Phys. Rev. Lett. 103, 161801 (2009).

[4] G. Bonvicini et al. (CLEO Collaboration), Phys. Rev. D 81, 031104 (2010).

[5] I. Adachi et al. (Belle Collaboration), Phys. Rev. Lett. 108, 032001 (2012).

[6] G. Bonvicini et al. (CLEO Collaboration), Phys. Rev. D 70, 032001 (2004).

[7] P. del Amo Sanchez et al. (BABAR Collaboration), Phys. Rev. D 82, 111102 (2010).

[8] K. F. Chen et al. (Belle Collaboration), Phys. Rev. Lett. 100, 112001 (2008).

[9] A. Bondar et al. (Belle Collaboration), Phys. Rev. Lett. 108, 122001 (2012).

[10] K. Seth, in Proceedings of Beautiful Mesons and Baryons on the Lattice, Trento, Italy, 2012, http://www.damtp.cam.ac.uk/research/hep/conferences/trentobeauty.

[11] S. Dobbs, Z. Metreveli, K. K. Seth, A. Tomaradze and T. Xiao, arXiv:1204.4205.

[12] A. Gray, I. Allison, C. T. H. Davies, E. Gulez, G. P. Lepage, J. Shigemitsu, and M. Wingate, Phys. Rev. D 72, 094507 (2005).

[13] S. Meinel, Phys. Rev. D 79, 094501 (2009).

[14] T. Burch, C. DeTar, M. Di Pierro, A. X. El-Khadra, E. D. Freeland, S. Gottlieb, A. S. Kronfeld, and L. Levkova et al., Phys. Rev. D 81, 034508 (2010).

[15] S. Meinel, Phys. Rev. D 82, 114502 (2010).

[16] R. J. Dowdall et al. (HPQCD Collaboration), Phys. Rev. D 85, 054509 (2012). 
[17] J. O. Daldrop, C. T. H. Davies, and R. J. Dowdall (HPQCD Collaboration), Phys. Rev. Lett. 108, $102003(2012)$.

[18] G. P. Lepage, L. Magnea, C. Nakhleh, U. Magnea, and K. Hornbostel, Phys. Rev. D 46, 4052 (1992).

[19] N. Brambilla et al. (Quarkonium Working Group), CERN Yellow Report, CERN-2005-05 arXiv:hep-ph/0412158. See Figure 4.10.

[20] R. Lewis and R. M. Woloshyn, Phys. Rev. D 84, 094501 (2011).

[21] R. C. Johnson, Phys. Lett. B 114, 147 (1982).

[22] C. T. H. Davies, K. Hornbostel, A. Langnau, G. P. Lepage, A. Lidsey, J. Shigemitsu, and J. H. Sloan, Phys. Rev. D 50, 6963 (1994).

[23] X. Liao and T. Manke, arXiv:hep-lat/0210030.

[24] J. J. Dudek, R. G. Edwards, N. Mathur, and D. G. Richards, Phys. Rev. D 77, 034501 (2008); J. J. Dudek, R. G. Edwards, M. J. Peardon, D. G. Richards, and C. E. Thomas, Phys. Rev. D 82, 034508 (2010); L. Liu, G. Moir, M. Peardon, S. M. Ryan, C. E. Thomas, P. Vilaseca, J. J. Dudek, and R. G. Edwards et al., arXiv:1204.5425.

[25] S. Aoki et al. (PACS-CS Collaboration), Phys. Rev. D 79, 034503 (2009).

[26] www.jldg.org.

[27] M. G. Beckett, B. Joo, C. M. Maynard, D. Pleiter, O. Tatebe, and T. Yoshie, Comput. Phys. Commun. 182, 1208 (2011).

[28] R. Lewis and R. M. Woloshyn, Phys. Rev. D 79, 014502 (2009).

[29] Belle Collaboration, I. Adachi et al., in Proceedings of International Workshop on Heavy Quarkonium 2011, Darmstadt, Germany, 2011 [arXiv:1110.3934].

[30] C. T. H. Davies, E. Follana, K. Y. Wong, G. P. Lepage, and J. Shigemitsu, Proc. Sci. LAT2007, 378 (2007) arXiv:0710.0741.

[31] M. G. Alford, T. Klassen and P. Lepage, Nucl. Phys. B, Proc. Suppl. 47, 370 (1996).

[32] C. Morningstar and M. J. Peardon, Phys. Rev. D 69, 054501 (2004).

[33] C. Gattringer and C. B. Lang, Lect. Notes Phys. 788, 1 (2010).

[34] T. C. Hammant, A. G. Hart, G. M. von Hippel, R. R. Horgan, and C. J. Monahan, Phys. Rev. Lett. 107, 112002 (2011).

[35] S. Godfrey and N. Isgur, Phys. Rev. D 32, 189 (1985).

[36] www.sharcnet.ca. 